\title{
Micro integration of optical components for the fabrication of active optical cables
}

\author{
F. Merchán \\ fernando.merchan@ziti.uni-heidelberg.de \\ D. Wohlfeld
}

\section{K.-H. Brenner}

\author{
ziti, Universität Heidelberg, Lehrstuhl für Optoelektronik, Mannheim, Germany \\ ziti, Universität Heidelberg, Lehrstuhl für Optoelektronik, Mannheim, Germany
}

Optical interconnects have become very interesting for short reach data transfer. We examine a new concept for integration and miniaturization of such systems based on recent experiments. The application of these designs is an active optical cable. The advantages of the concept and the employed technologies are presented. [DOI: 10.2971/jeos.2010.10056]

Keywords: optical interconnects, micro-coupler, micro-mirror, active optical cable

\section{INTRODUCTION}

Due to the high bandwidth, lower power consumption and better signal to noise ratio, optical interconnects have become attractive also for the short distance region. The complexity of the alignment requirements and the increased costs for fabrication, however, discourage the application of optical interconnects in this regime.

Additional focusing optics and active adjustment can be eliminated by constructing a fully integrated, self guiding microoptical system. For the master fabrication, a standard HSC (high speed cutting) machine may achieve $\mu \mathrm{m}$ precision and additional finishing methods enable optical surface qualities.

In our application, the active optical cable, we combine high speed electronic connectors with optical fibers for the high bandwidth transfer of large data volumes. The signal conversion is performed in the connector through an optical coupler and optoelectronic conversion.

\section{DESIGN OF THE OPTICAL COUPLER}

An optical system based on plastic replication of negative metal moulds has been designed. The versatility and high accuracy of micro machining is utilized to construct a self guiding and adjusting mechanism for the optical fiber. The monolithic integration of mirror, funnel, fiber-guide and mount reduces alignment complexity compared to many other coupling methods.

The replication of a metal mould ensures the optical quality of the mould in the plastic substrate making it a very accurate fabrication method, attractive also because of its low cost.

In the structure, a micro mirror is used to redirect the beam from the laser into the fiber, positioned $90^{\circ}$ relative to the laser. The self guiding structure for the fiber is equipped with a funnel structure (Figure 1) which gets thinner when getting closer to the light source [1]. The coupler is mounted at the same plane as the electronic devices placed directly under the substrate (Figure 2). Important to note is the need of a reflective coating of the mirror.

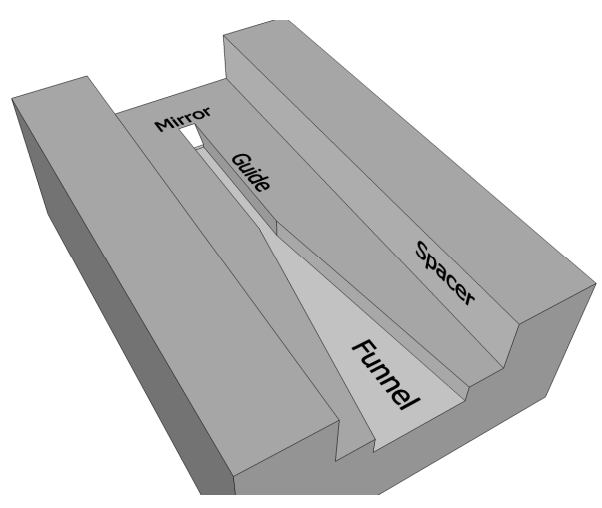

FIG. 1 Model of the replicated structure.

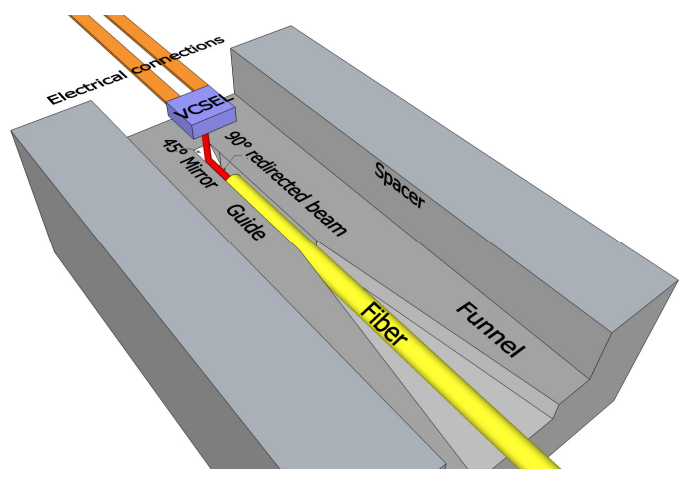

FIC. 2 System with optical and electronic devices. PCB blinded out. 


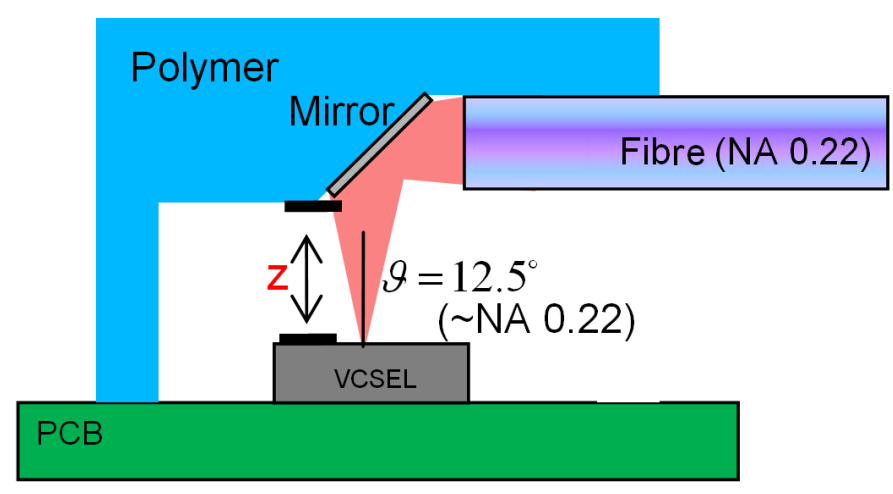

FIG. 3 Simplified model of the coupling structure.

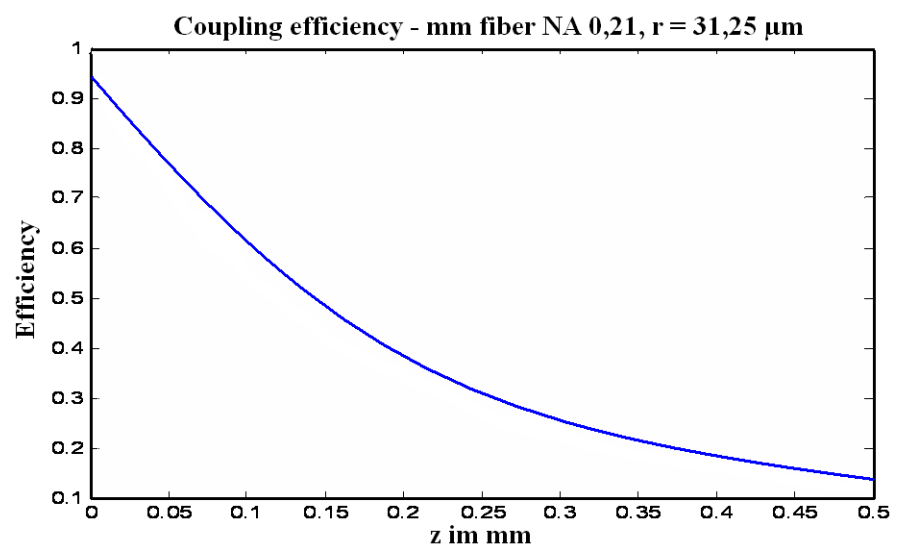

FIG. 4 Distance dependant coupling efficiency.

\section{SIMULATION OF THE OPTICAL COUPLER}

For the simulation of the optical-coupler, we developed a customized ray-trace software, which enables a threedimensional design and takes the fiber parameters into consideration. With this software, we calculated the coupling efficiency depending on the separation between laser and fiber [2]. A simplified model of the system is shown in Figure 3. Figure 4 shows the result and demonstrates the importance of minimizing the distance between VCSEL and fiber. By varying the distance from $z=0 \mu \mathrm{m}$ to $z=200 \mu \mathrm{m}$, the coupling efficiency reduces from $95 \%$ to roughly $40 \%$.

\section{FABRICATION}

The fabrication of the coupler is a two step procedure that begins with the fabrication of the metal mould using a standard HSC (high speed cutting) machine. The methodology employed for the manufacture of the mould is based on a tolerance analysis of fabrication errors caused by limitations of the employed machine and on a compromise to minimize fabrication costs.

CAD-models of the coupler were created and exported to the HSC machine to manufacture the mould of Figure 5. The funnel- and guiding-structures were designed for a $125 \mu \mathrm{m}$ diameter optical fiber, as shown in Figure 6 for a single channel.

The replication of the mould is performed using UV-curable

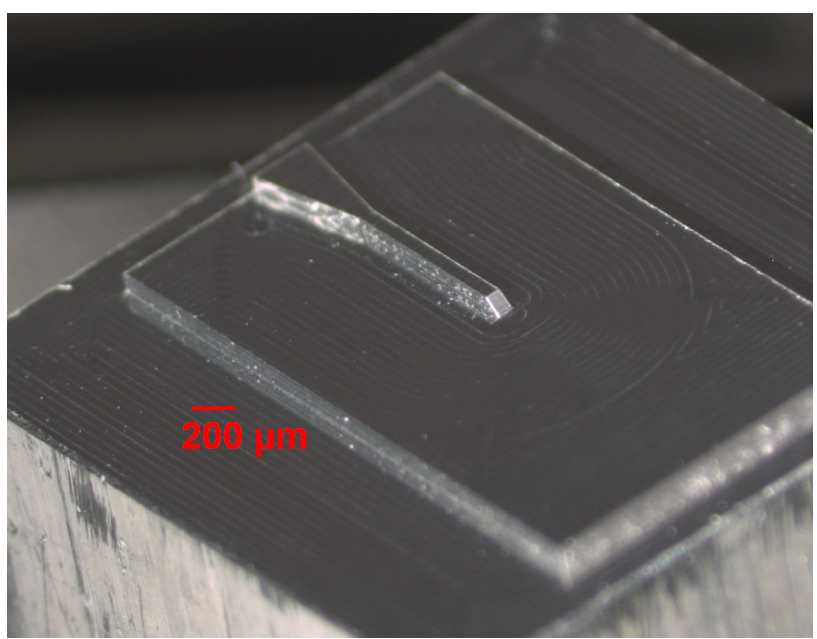

FIG. 5 Fabricated metal mould.

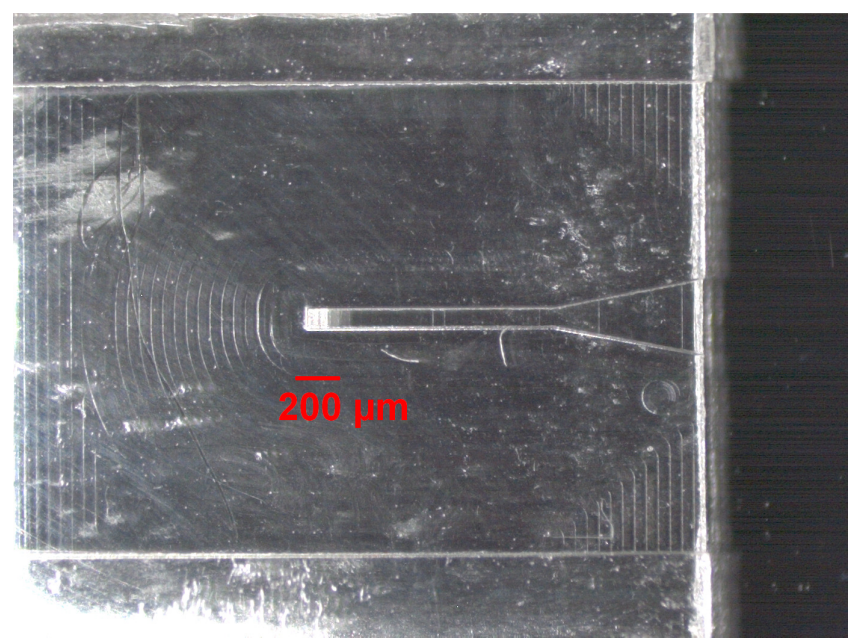

FIG. 6 Plastic replication.

polymer [3]. First the mould is filled up with the polymer in liquid form and afterwards cured with UV-radiation. Finally, the mold and the replica are separated. The surface of the mirror has to be sputtered with a reflective coating in order to improve the reflectivity (Figure 7).

\section{QUALITY OF THE SURFACES AND ADVANTAGES OF THE FABRICATION METHOD}

There are several advantages of using diamond turned metalmoulds compared to other lithographic techniques. They are first, the 5 degrees of freedom of the HSC with high accuracy; second, the possibility to finish the mirroring surfaces in order to reach optical quality; and third, the durability of the metal master since it is replicated with much more flexible materials. In Figures 8 and 9, measurements of the surface roughness of the metal-mould are shown. The mirror's surface (area three) was finished and presents a roughness two orders of magnitude better than those which were not finished (areas one and two). 


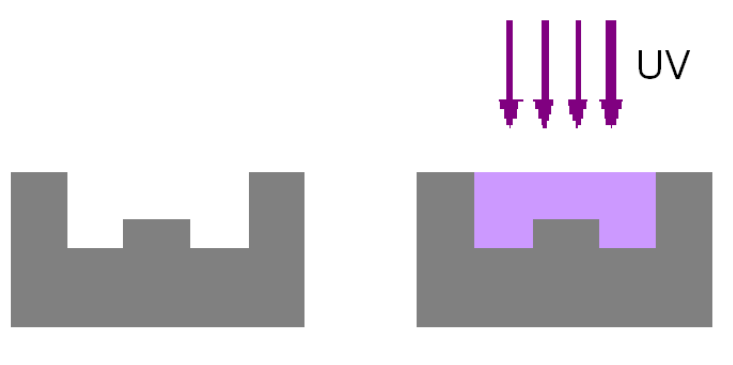

Negative metal mould

Curable polymer

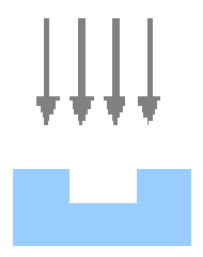

Separation

FIG. 7 Replication process with UV-curable polymer.

\section{APPLICATION: THE ACTIVE OPTICAL CABLE}

The most important advantages of this construction for the fabrication of optical cables are first, the capability of parallelizing many channels working at current speeds, i.e. $5 \mathrm{Gbps}$, to reach very high speed communication rates, and second the miniaturized construction of a low-cost multi-channel connector. The centring of the VCSEL to the optical axis of the fiber is achieved by a passive alignment of the fibers and an active alignment of the complete fiber group. The use of surface emitting lasers (VCSEL), because of its small size, enables very compact constructions.

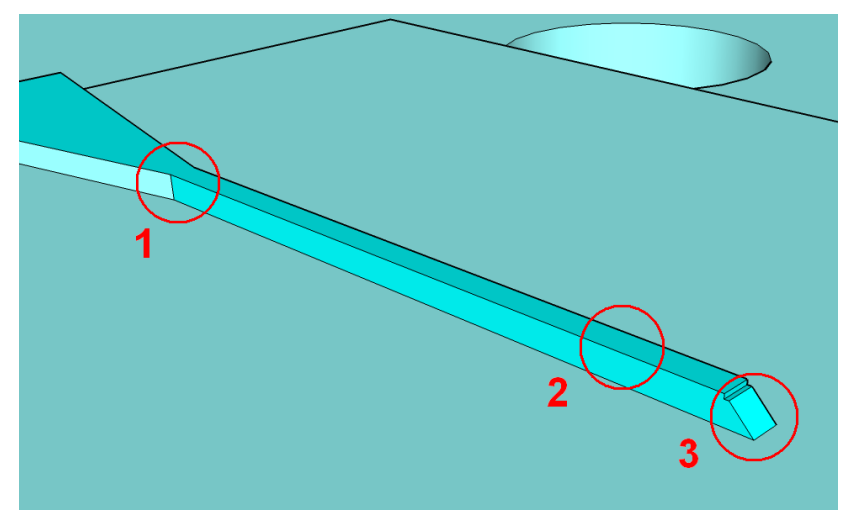

FIG. 8 Measured points of the metal mould.
Our design minimises the distance between the light source and fiber front (Figure 10) to $150 \mu \mathrm{m}$. The multi-channel system is made by parallelising the single coupler on a $250 \mu \mathrm{m}$ pitch to meet wafer fabrication standards. Thus, it is possible to obtain 12 channels working at 5 Gbps each, reaching a bandwidth of $60 \mathrm{Gbps}$ in a width of just $3 \mathrm{~mm}$. On a single PCB we combine the optical coupler, electronic drivers for the laser diodes and the electrical connections as shown in Figure 11.

\section{FIRST MEASUREMENTS: THE EYE DIAGRAM}

The block diagram of the system for first proof of principle tests is shown in Figure 12. The high speed signals are generated by a FPGA provided with a high speed sender on the same chip. The high speed output is connected directly to the laser driver. The critical path starts with the wire connections between the laser-driver and the laser (VCSEL). For the first design, we added some additional flexibility, which made the connecting wires intentionally very long (some millimetres), sacrificing quality of signal at the diode.

In Figures 13 and 14, we show the measured eye diagrams at the points shown in Figure 12 for the signal measured near the driver and the driving signal for the VCSEL. Because of the long paths between driver and laser-diode the signal is no-
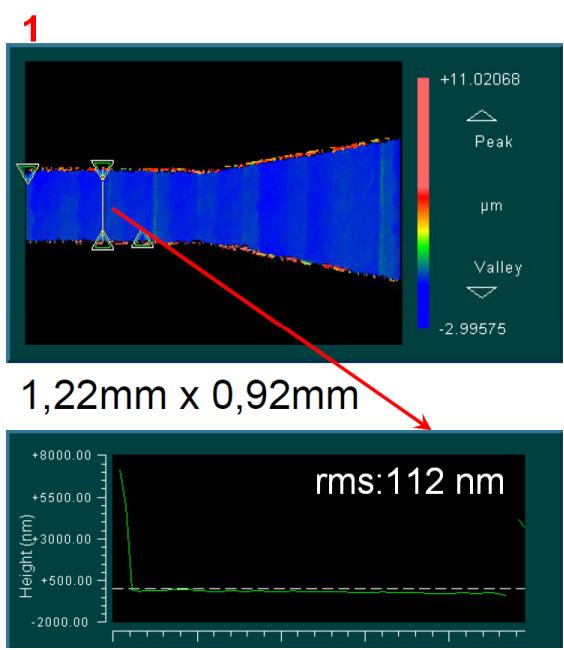

2

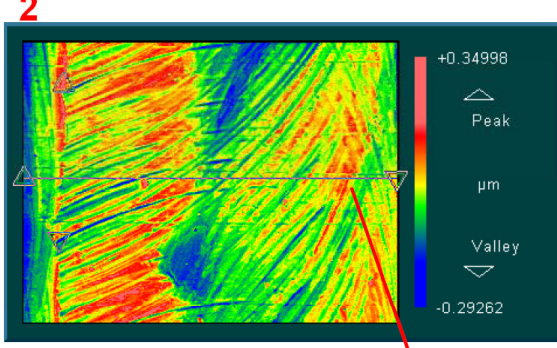

$0,15 \mathrm{~mm} \times 0,11 \mathrm{~mm}$

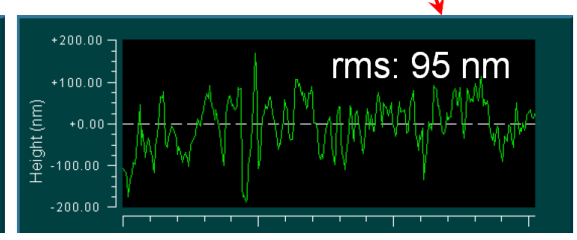

3
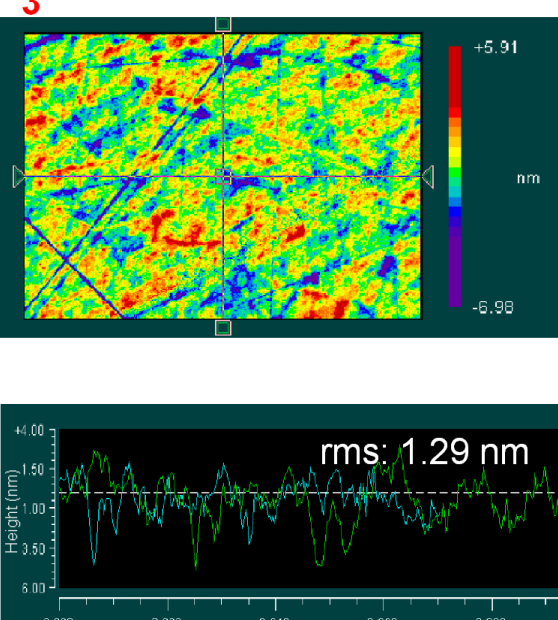

FIG. 9 Roughness measurements of the metal mould. Picture from Aalen University. 


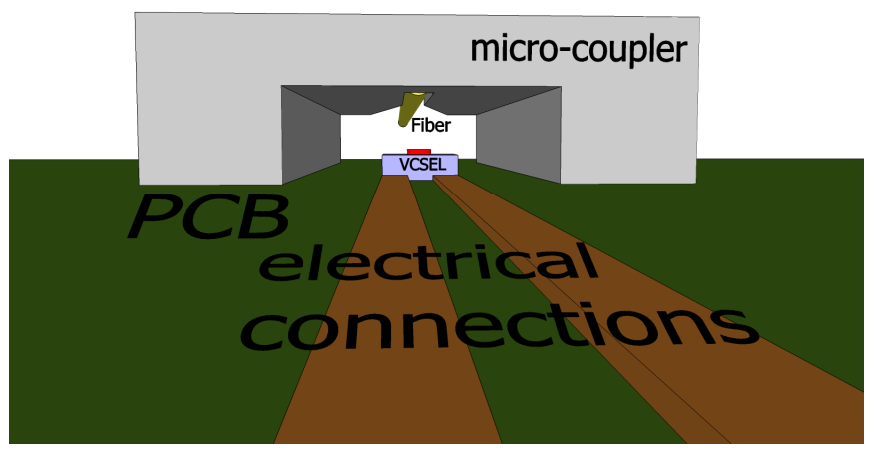

FIG. 10 Side view of the single channel integrated system.

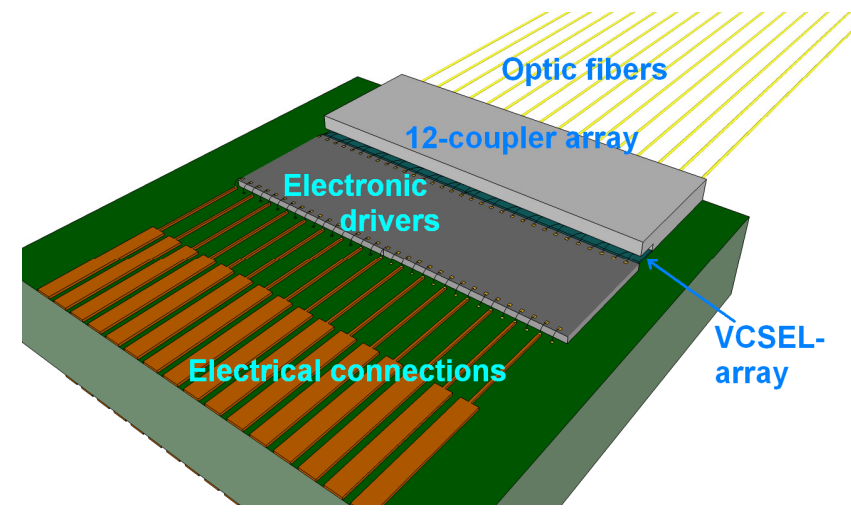

FIG. 1112 channel integrated system.

tably degraded. In a second design, we will remove the flexibility, which should result in much better signal quality.

\section{CONCLUSIONS}

We present a concept for active optical cables based on micromilling for the manufacture of optical devices. The concept has many advantages not only with respect to handling, mounting and adjustment procedures but also because it is a low cost solution in a compact package, simplifying assembly and adjustment procedures.

The design is not restricted to active optical cables but can be used in any optoelectronic diode-fiber alignment scenario.

Standard HSC machines represent a very accurate low cost method for the fabrication of metal moulds with optical quality and by replicating the structure in a plastic substrate, low cost micro-couplers can be manufactured.

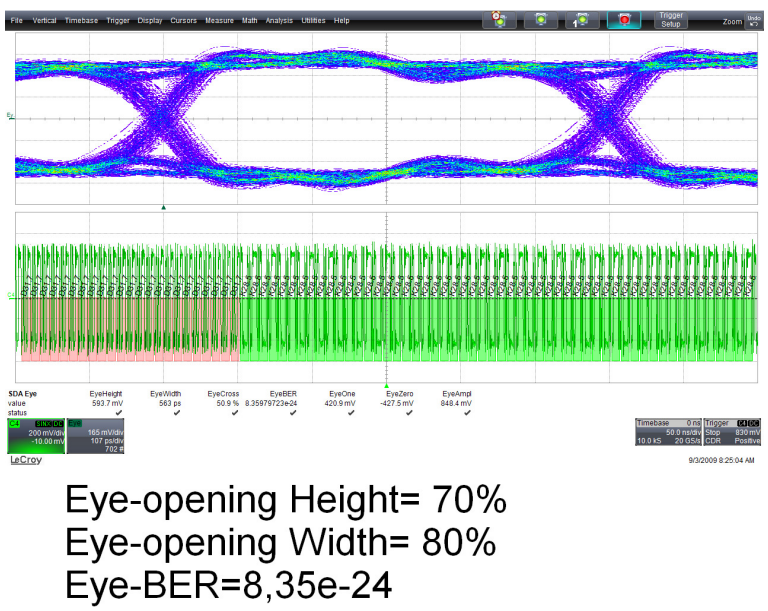

FIG. 13 Electrical signal at VCSEL driver's input.

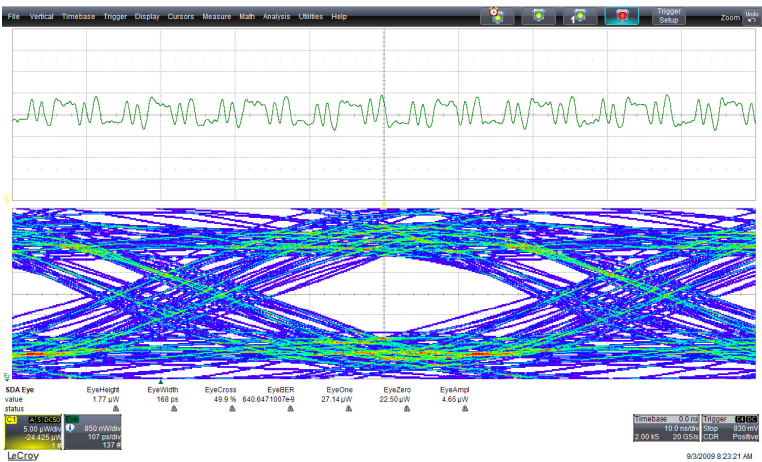

Eye-opening Height $=40 \%$

Eye-opening Width $=52,5 \%$

Eye-BER=0,64e-6

FIG. 14 Eye diagram of the optical signal

\section{ACKNOWLEDGEMENTS}

Authors acknowledge Prof. Dr. Börret, Prof. Dr. Berger, Dr. Georg, and Prof. Brüning for their contribution.

\section{References}

[1] D. Wohlfeld, and K.-H. Brenner, "Fabrikation und Integration von mechanisch-optischen Strukturen zur Kopplung von VCSELs mit Glasfasern" in Proceedings of Mikrosystemtechnik Kongress 167169 (Mikrosystemtechnik Kongress, Freiburg, 2005).

[2] D. Wohlfeld, Simulation, Analysis, and Fabrication of Miniaturized Components with Applications in Optical Interconnects and Parallel Microscopy (PhD thesis, University of Heidelberg, 2009).

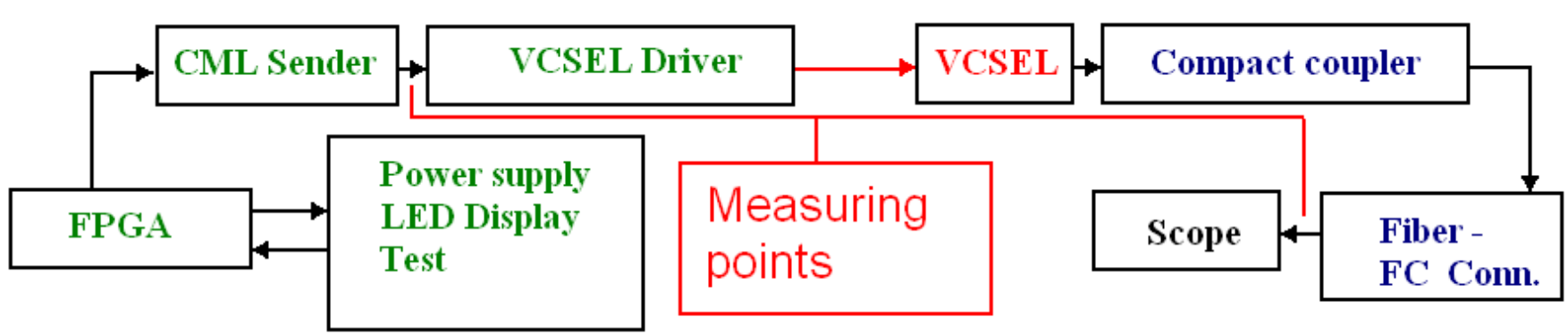

FIG. 12 Block diagram of the test system. 
[3] K.-H. Brenner, M. Kufner, S. Kufner, J. Moisel, A. Muller, S. Sinzinger, M. Testorf, J. Göttert, and J. Mohr, "Application of threedimensional micro-optical components formed bylithography, electroforming and plastic molding" Appl. Opt. 32, 6464-6469 (1993). 